\title{
Social and Epidemiological Research on Alcohol: Research Presented at Meetings of the Kettil Bruun Society between 1983 and 2017
}

\author{
Henk Garretsen ${ }^{1}$, Ien van de Goor ${ }^{1}$, Diana Roeg ${ }^{1,2}$ and Dike van de Mheen ${ }^{1}$ \\ ${ }^{1}$ Tranzo, Tilburg School of Social and Behavioral Sciences, Tilburg University, The Netherlands \\ ${ }^{2}$ Kwintes Supported Housing and Shelter Research Program, Tilburg University, The Netherlands
}

\begin{abstract}
Aims: The Kettil Bruun Society (KBS) is a leading international society, known in full as the Kettil Bruun Society for Social and Epidemiological Research on Alcohol. This paper aims to analyse research presented at KBS annual meetings and provide an overview of the developments in the research agenda in the field. In this way we try to gain insight into worldwide developments in the research agenda on social and epidemiological research on alcohol between 1983 and 2017.
\end{abstract}

Methods: For the period 1983-1992, all annual meetings were the object of study. For the period after 1993, three sample periods of the annual meetings were used. The current paper is the fourth overview paper in a series. All papers have been reviewed with regard to their content, the research methods used, the country of origin, and the gender of the presenting authors.

Results: The number of papers presented at the annual KBS meetings increased from 35 in 1983 to over 160 in the years after 2009. In the period 1983-2017, the percentage of papers on policy and prevention doubled. Surveys have been the most popular research method in the period reviewed here.

Conclusions: The KBS is the international society for this type of research, and developments within the KBS give an indication of the worldwide developments in the research agenda on social and epidemiological research on alcohol between 1983 and 2017.

\section{Introduction}

In order to study worldwide developments in the research agenda on social and epidemiological research on alcohol between 1983 and 2017, papers presented at the annual meetings of the Kettil Bruun Society for Social and Epidemiological Research on Alcohol (KBS) have been reviewed. KBS is the international society for this type of research (Demers, Garretsen, Room, Rossow, \& Ugland, 2004). KBS is an independent society open to all scientists working in the field. KBS is the successor of the epidemiology section of the International Council on Alcohol and Addictions. In 1987 this section joined the International Group for Comparative Alcohol Studies to establish the Kettil Bruun Society, named after one of the pioneers in social alcohol research. The core activity of KBS is a five-day annual meeting.

Research presented at these annual meetings gives a good overview of the developments in the research agenda in the field. The current paper is the fourth overview paper in a series. In 1993, a paper was presented in which the developments in the annual meetings in the period 19831992 were described (Garretsen, van Oers, \& van de Goor, 1993). Two follow-up papers have been presented in 2003 and 2011 (Garretsen, Bongers, van de Goor, \& Roeg, 2003; Garretsen et al., 2012).

\section{Method}

\section{Annual Meetings and Papers Included in the Study}

For the period 1983-1992, all annual meetings were the object of study. For the period after 1993, three sample periods of the annual meetings were used because of the very large increase in the number of papers presented. The host country/city and the continent of the annual meeting is of influence on the number of participants and their country of origin. In that way it also influences the number and content of the papers presented. In the previous papers, results were presented for the years 2002/2003 and 2009/2010, and for

Correspondence: Prof. Dr. Henk F.L. Garretsen, Tranzo, Tilburg School of Social and Behavioral Sciences, Tilburg University, PO Box 90153 , 5000 LE, Tilburg, The Netherlands. Telephone: + 31 (0)13 466 3299/2969, Email: H.Garretsen@ tilburguniversity.edu

Financial support: No external funding

Declaration of interest: None

Keywords: alcohol research, Kettil Bruun Society, alcohol epidemiology 
the period 1983-1992. This paper adds results from 2016 (Stockholm) and 2017 (Sheffield). The authors feel these years are comparable to each other with regard to the geographical position of the host cities (Europe).

All papers have been reviewed with regard to their content, the research methods used, the country of origin, and the gender of the presenting authors. For the period 1983-1992, the review included the whole paper; for other time periods, the review included either the whole paper or the abstract only. Pre-symposia were often held and focused on specific issues. Papers presented exclusively at these pre-symposia have not been included, but papers that were presented both at pre-symposia and at the main symposia are included.

\section{Review Process}

For this paper, all abstracts and, if needed, the full papers were reviewed by two of a team of four researchers (the authors of this paper), independent of each other. When the two judgments did not match, the paper was discussed by the two researchers involved in order to reach consensus. In case of different opinions, the final judgment was given by the principal investigator (HG). All researchers are experienced in the field of social and epidemiological research on alcohol and all have participated in a number of annual KBS meetings.

\section{Measurements}

The measurements in this paper are similar to those used in the first three manuscripts (Garretsen et al., 1993; 2003; 2012). To compare all KBS papers from 1983 onwards, it was necessary to use the same categories and criteria that were used in the first paper (Garretsen et al., 1993). In the last two manuscripts some small additions are made (see below).

Twelve categories were used to describe the content of the papers presented at KBS meetings: drinking patterns, drinking problems, methodology, explanatory models, alcohol and suicide, treatment and care, self-help groups, policy and prevention, norms and attitudes, women and alcohol, historical studies, and alcohol and drugs. Each paper was labeled with one of these categories. When the content could be assigned to two or more categories, the general rule was to choose the category that in general was the least frequently scored. This was decided in 1993 and the same procedure has been used since. For example, if a paper covered both drinking patterns and drinking problems, the paper was scored in the less frequently scored category of drinking problems. In the years 2016 and 2017 a thirteenth category was added: ethical issues (e.g., funding by the alcohol industry), since it was felt that this topic was becoming more prominent.

The following categories were used to describe the research methods used in the papers: surveys, existing data sources, qualitative methods/interviews, literature studies/reviews, and "other." In the first two review papers (Garretsen et al., 1993; 2003), the category "other" was scored when the method used was unknown/not described, or when papers described multi-method studies. Later on the category "multi-method studies" was added as a separate category next to the category "other" in order to be able to describe how many papers fell into this category (Garretsen et al.,
2012). For this category it is not possible, however, to make a comparison over all the years.

The country of origin and gender of presenting authors were registered. The country of origin was determined by the country of research affiliation of the first author involved. In the 1993 paper, countries were geographically grouped using the following categories: United States and Canada, Nordic countries (Norway, Sweden, Finland, Denmark, and Iceland), United Kingdom and Ireland, Mid-Europe (Netherlands, Germany, Switzerland, and Austria), Southern Europe (France, Italy, Spain, Portugal), and Eastern Europe (Hungary, Poland, Czech Republic, Yugoslavia [1993] and Croatia, Bosnia, and Herzegovina [post 1993], Lithuania, and Russia), and "other." In those days most participants came from Europe and the United States or Canada. This situation, however, has changed to a large extent. For this reason, for 2009-2010 and 2016-2017 the category "other" was replaced by the categories Australia/New Zealand, South and Central America, Asia, and Africa to get a better idea of the geographical spread of the presenting authors. However, to enable comparison with previous years, these countries were grouped as "other".

\section{Results}

The number of papers presented at the annual KBS meetings increased substantially between 1983 and 2009 and remained roughly the same in the years after. In 1983, 35 papers were presented; in the years 2009-2010 and 20162017, between 160 and 185 papers were presented (Figure 1)].

\section{Figure 1}

Absolute number of papers presented at KBs 1983-2017

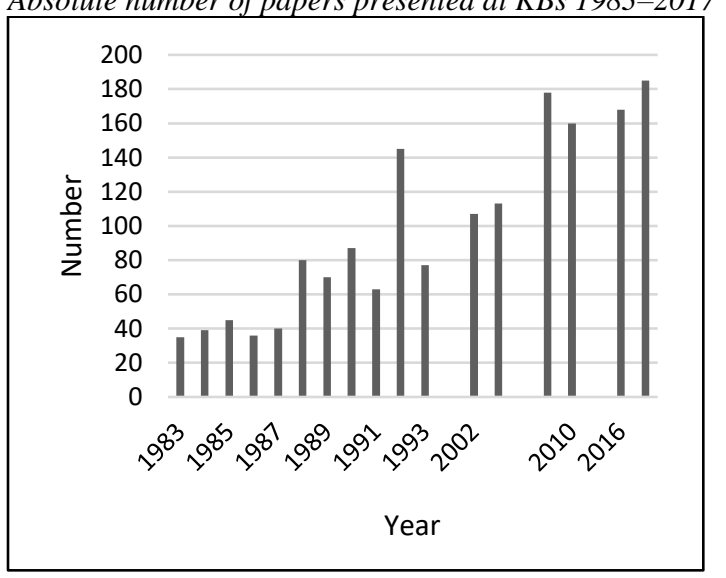

Content: Increasing Attention for Research on Policy and Prevention.

Both in Stockholm (2016) and Sheffield (2017) a quarter of all papers dealt with "policy and prevention," followed by the categories "alcohol problems" and "drinking patterns" (Figure 2). Of course, many fluctuations have taken place during the years, as indicated in the eight categories most frequently scored during the whole period (Figures $3 \mathrm{a}$ and $3 \mathrm{~b}$; for clarity, results are divided over two figures). The percentage of papers on policy and prevention has doubled from, very roughly, an average of about $12 \%$ in the years 1983-1993 (Garretsen et al., 1993), to almost 25\% in 2016 
and 2017. In the earlier days of KBS, relatively more papers dealt with drinking patterns.

A number of topics were only prevalent in a limited period (Garretsen et al., 1993; 2003; 2012; results for these topics are not shown). Between 1990 and 1991, for instance, the topic "self-help groups" was relatively prevalent. In 1991 more than $10 \%$ of the papers dealt with this topic. In roughly the same period about $5 \%$ of the papers were about "alcohol and suicide." In 1984, the topic "women and alcohol" was very popular; $17 \%$ of the papers fell into in this category. In subsequent years, this was the case for just under $5 \%$ of the papers: in $20164 \%$, and in $20172 \%$. In 1985-1986, 10-15\% of the papers were categorized as "historical studies," while later on, very few were classified in this way. In 2016 and 2017 , we checked whether the category "ethical issues" gained attention. However, in 2016, just one paper clearly dealt with this topic, and in 2017 , four papers.
Figure 2

Content of papers presented at KBS Stockholm (2016) vs KBS Sheffield (2017)

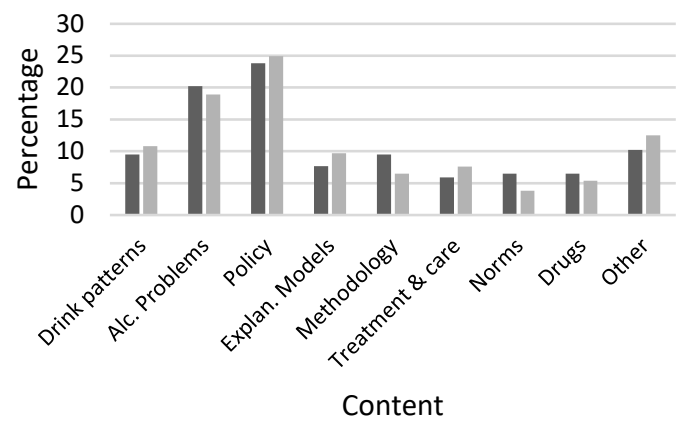

—Stockholm (2016) Sheffield (2017)

\section{Figure 3a}

Content of KBS papers presented, 1983-2017

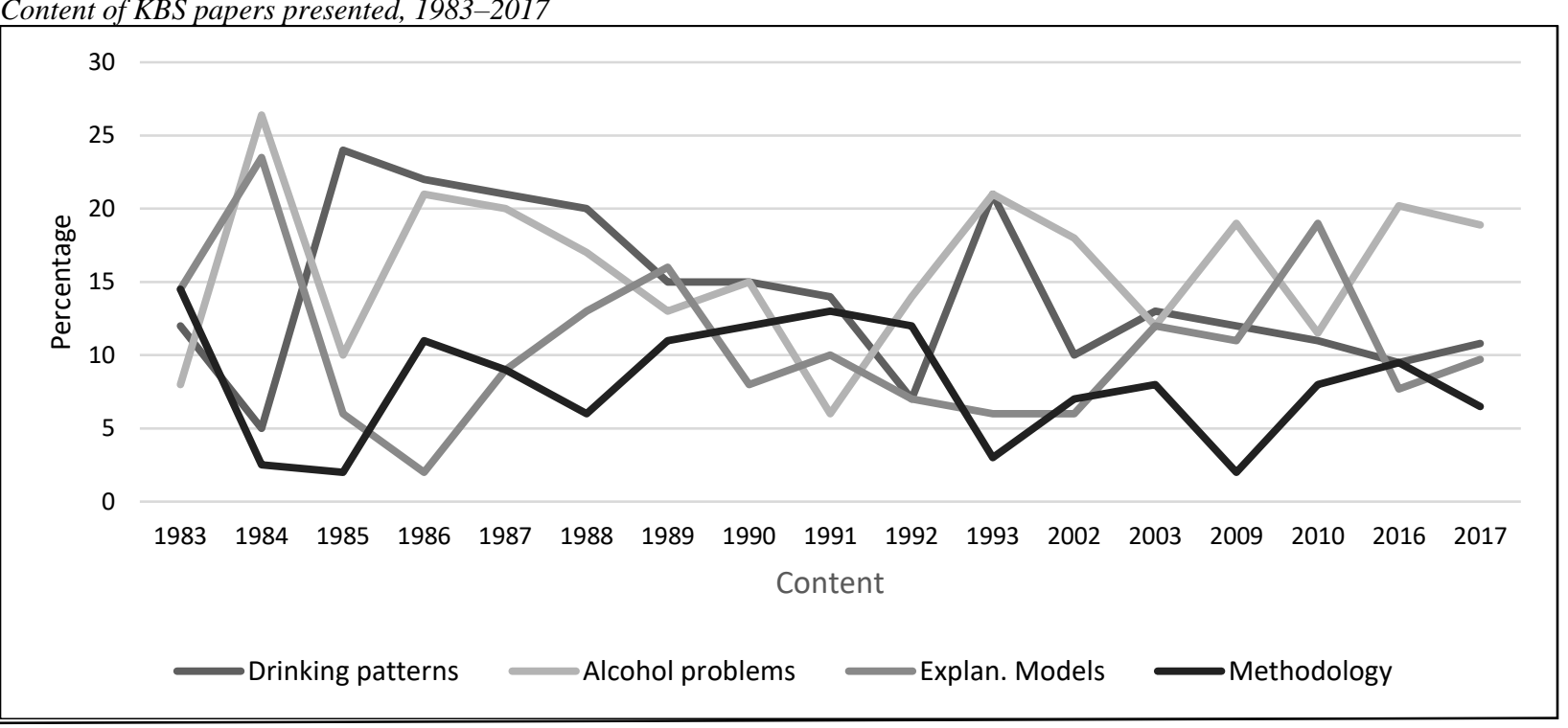

Figure 3b

Content of KBS papers presented, 1983-2017

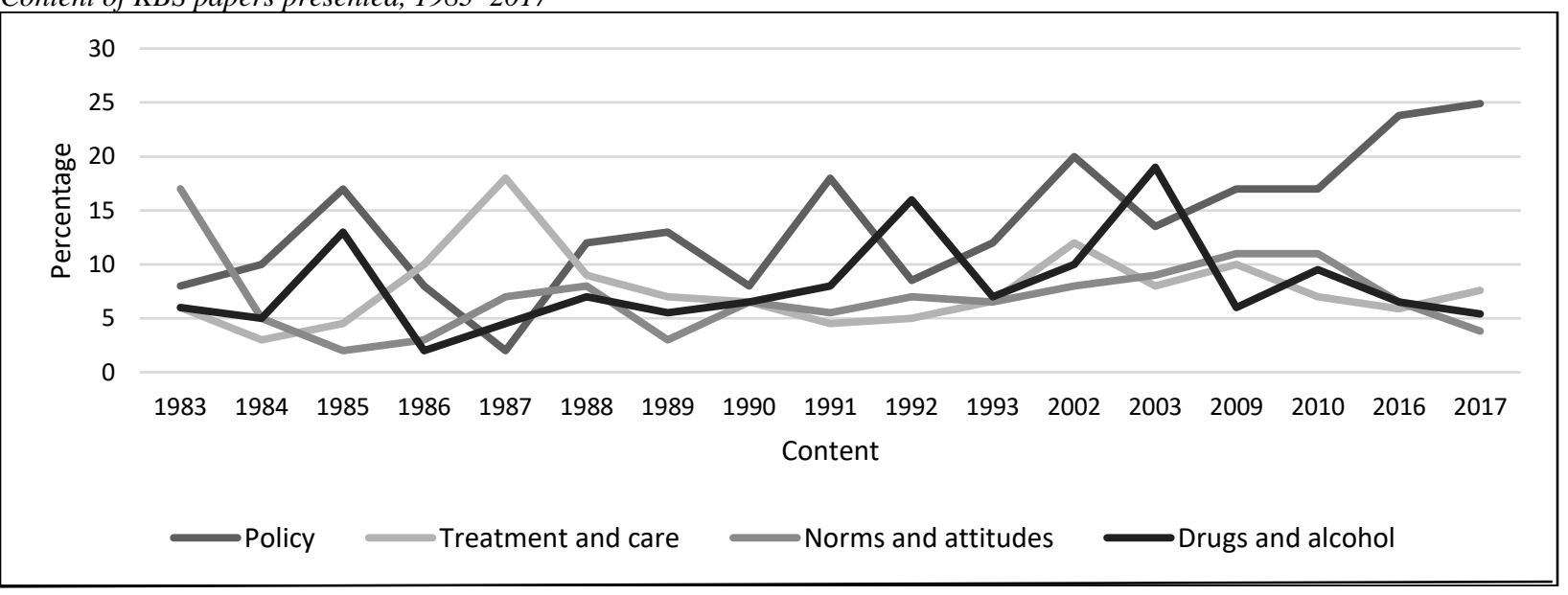




\section{Research Method: Surveys Used Most in All Years}

By far surveys were the most popular research method used in all sample periods. About $40 \%$ of the papers were based on surveys (Figures 4 and 5).

\section{Figure 4}

Research methods used in papers presented at KBS Stockholm (2016) and KBS Sheffield (2017)

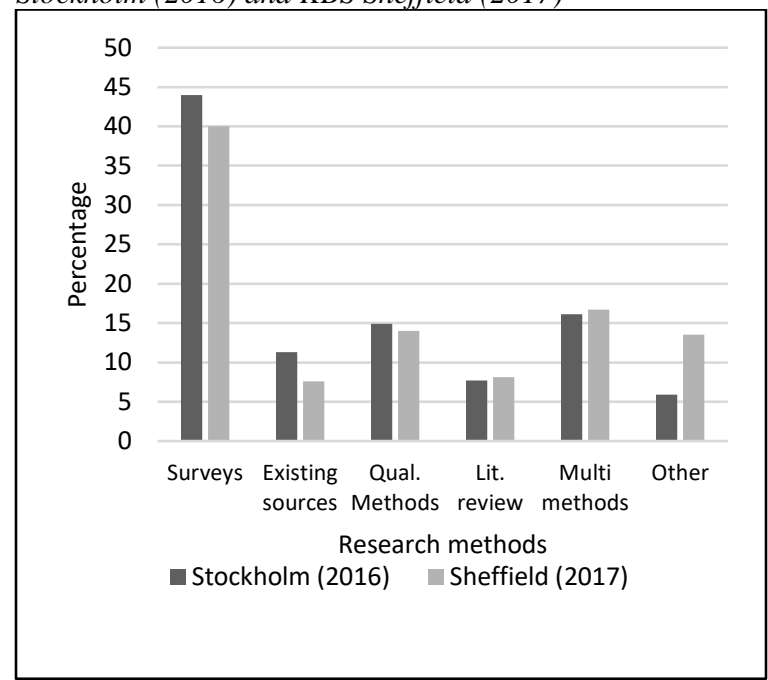

Nowadays multi-method studies appear to be more popular. In 2016 and $2017,16-17 \%$ of the papers were classified as such (Figure 4). In 2009-2010 this was the case for 11-12\% (results not shown). During the first two periods, however, we cannot tell, because the category "multi-method studies" was not used in the studies from these periods.

In $2016-2017,14-15 \%$ of the papers were based on "qualitative methods/interviews," with this percentage lower than in 2009-2010 but still a little higher than in the years before (Figures 4 and 5). Literature review papers were more

\section{Figure 5}

Research methods used in papers presented at KBS in 19832017

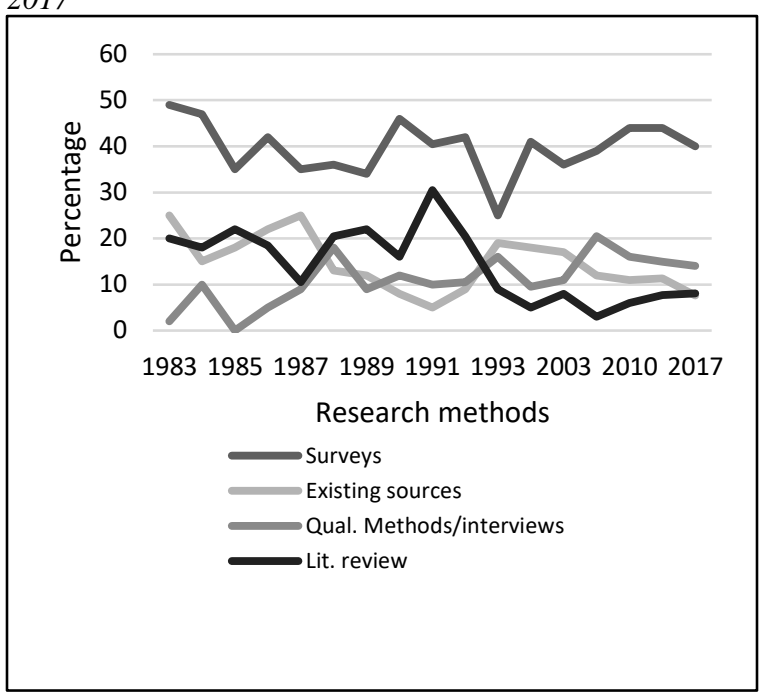

common between 1983 and 1993. In 2016-2017 about 8\% of papers fell into this category (Figures 4 and 5).

\section{Participants: Increase till 2009-2010, Decrease} Afterwards

With ups and downs, the number of participants has on average increased from 1986 onwards, but compared to 2009-2010, the number of participants decreased in 20162017 (Figure 6). The highest numbers were reached in 1992 in Toronto (184), in 2009, in Copenhagen (243) and in 2010 in Lausanne (212).

Figure 6

Number of men and women attending KBS meetings, 1983-2017

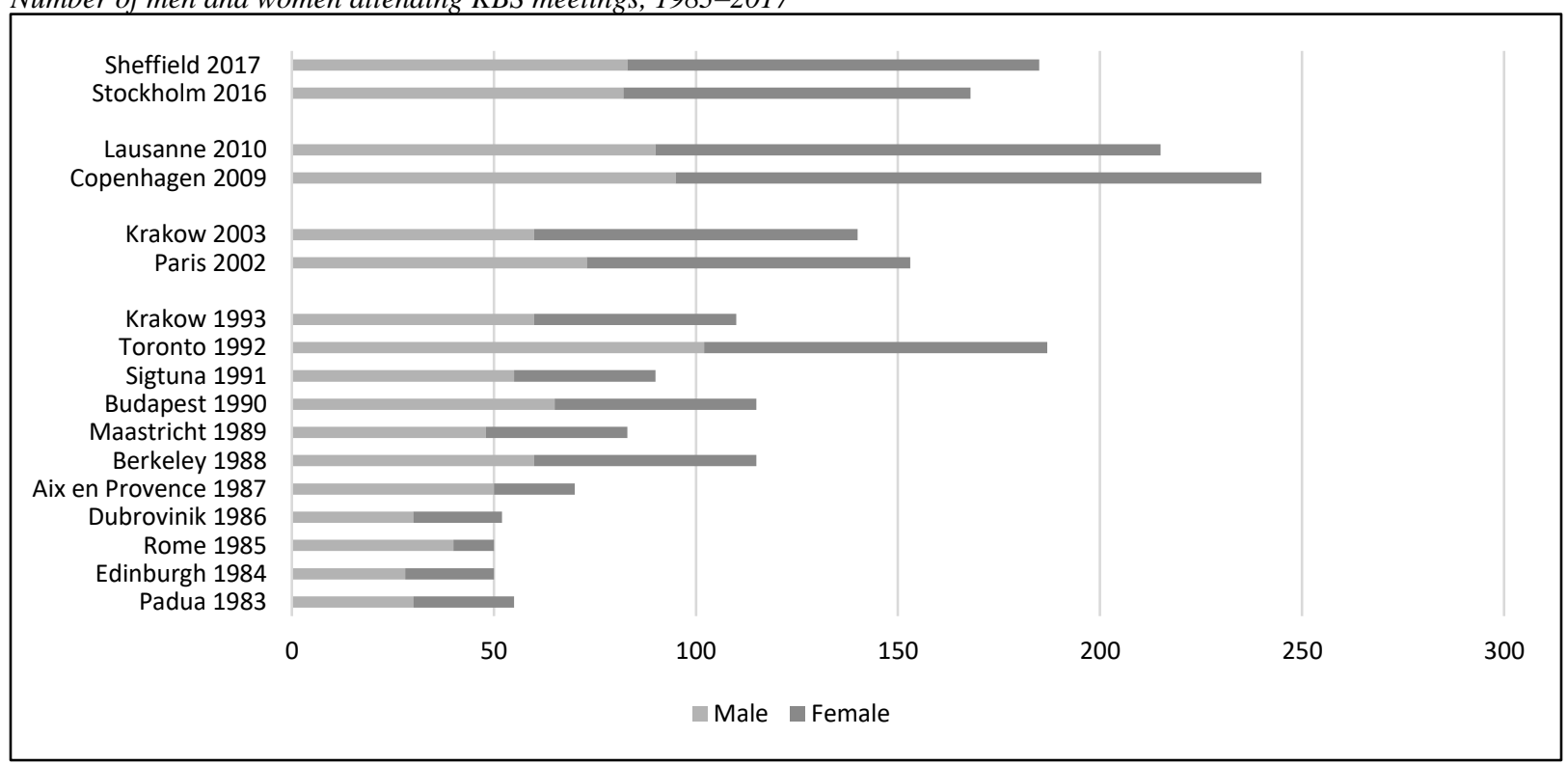


The percentage of female participants increased considerably from about one-quarter to one-third in 1983 1987 to about $40-50 \%$ in $1988-1993$. In $2002-2003$ this further increased to about 55\%, and again in 2009-2010 to approximately $60 \%$. The percentage has dropped a little in 2016-2017 (50\% and 55\%, respectively).

\section{Region of Origin: Growing Geographical Spread}

In the years 1983-1993, almost all participants came from North America (United States and Canada) and Europe, especially the Nordic countries (Figure 7). In 1983 more than half of the participants came from just two countries: the United States and Finland.

\section{Figure 7}

Number of participants of KBS meetings grouped by geographical region, 1983-2017

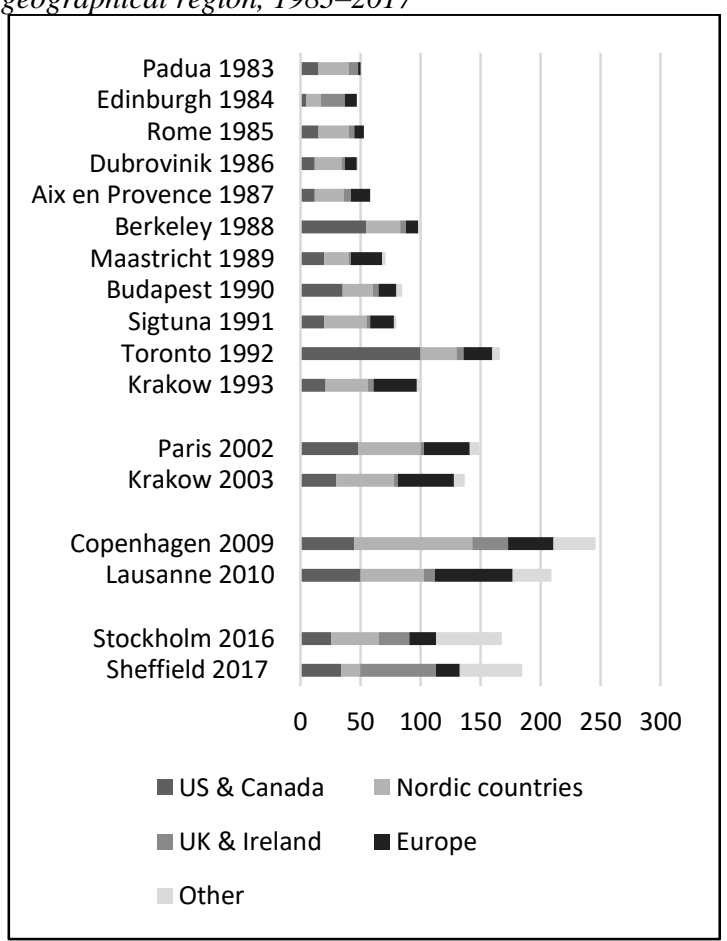

Also in 2002, 2003, 2009, and 2010, most participants came from North America (to a somewhat lesser degree) and from the Nordic countries. (Relatively more from Sweden and less from Finland). In the last years the contribution of North America and the Nordic countries is somewhat decreasing. It is remarkable that since 20092010 all continents are represented. For the years 20092010 and 2016-2017 this is shown in Figure 8, in which the category "other" is specified. Participants come nowadays also from Australia and New Zealand, Asia, Africa, and South and Central America.

\section{Discussion}

This paper describes characteristics of papers and participants at the annual meetings of KBS. KBS is the international society for this type of research and developments within KBS may give an indication of the worldwide developments in the research agenda on social and epidemiological research on alcohol between 1983 and 2017.

Figure 8

Number of participants at KBS meetings grouped by geographical region, 2009-2017: specification of "other" geographical regions

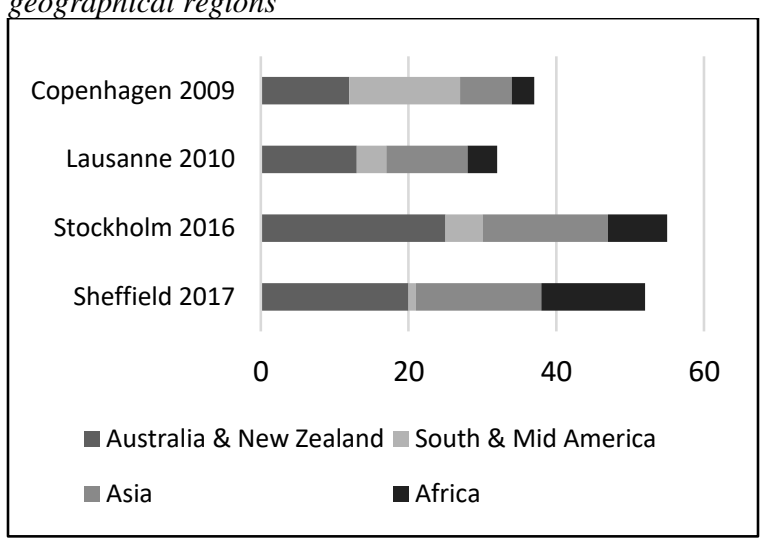

With regard to the content of the papers it is notable that in the earlier days of KBS, relatively more papers dealt with drinking patterns. This is perhaps not that surprising: in that time there was no big tradition in social (and epidemiological) alcohol research, and it makes sense to start with mapping the phenomena as such. Over the years the percentage of papers on policy and prevention has doubled, comprising almost one quarter of all papers presented in 2016-2017. It would be interesting to study how the researchers involved in these papers are employed; perhaps they are relatively more employed by (local) governments and state institutions, and studying and evaluating policy and prevention is part of their daily work.

A number of topics, for instance, "alcohol and suicide" and "self-help groups," were more prevalent during a limited number of years. This may be explained by the fact that international collaborative projects and pre-symposium meetings focusing on these topics took place during these years. Quite often pre-symposia were focused on a specific issue. For many years this has been the case for the topic "gender and alcohol." Quite often papers presented in the pre-symposia were also presented at the main symposium; papers only presented at these pre-symposia have not been included in the analysis. For the years 2016 and 2017, the category "ethical issues" has been added. In 2016 just one paper clearly dealt with this topic; in 2017 four papers. This category was added because it was thought (on the basis of plenary debates and collegial discussions at KBS meetings) that this topic had aroused more attention in recent years.

Surveys have been the most used research method over the years: $40 \%$ of all papers were based on surveys. Methods have become more varied over the years. The percentage of multi-methods studies has increased. This topic was not included as a separate category in the first two sample periods because of the low prevalence. In 2016 and 2017 one sixth of the papers was classified as such. This means it is worthwhile to include multi-method studies as a separate category. 
The number of participants in 2016-2017 was somewhat lower than in 2009-2010. This does not necessarily mean that the attention for this type of alcohol research is decreasing. A possible explanation could be that less funding to attend the meetings is available. Notably, nowadays researchers come from all continents. However, the number of participants from other continents is not always big and "stable". Probably the lack of sufficient funds for researchers in low- and middle-income countries is a reason for this.

The geographical location of the host city is indeed an important factor. For instance, in 2017 in Sheffield the number of participants from the United Kingdom and Ireland was very high.

It is felt that the results still give a good indication of the global developments in the research agenda on social and epidemiological research on alcohol, but the results should not be interpreted in a more than general way. Many limitations are evident, and they have grown in importance over the years. First, the raw data from the early years are not available anymore - this means that data as presented in the earlier papers has been used. New and/or secondary analyses on these older data are unfortunately not possible.

Second, in order to compare the papers over time, the categories as used in the first paper had to be used. In this way it is possible to study whether "old" topics still are addressed, but "new" trends are more difficult to detect. For instance, the category "multi-method studies" was not used in the first two papers. And there were no separate categories for experimental designs, randomized controlled trials, and implementation studies. If the use of research methods would be studied again, categories like these should be added. In addition, overlaps between categories, such as explanatory models and categories like "alcohol problems," are difficult to assess.

It seems not possible to find a solution for this problem. In the first papers, when a topic could be assigned to two or more categories, it was decided to choose the category that in general was the least frequently scored. This had to be continued in the present analysis; however, it seems to be an arbitrary choice. Of course this is a major limitation. For the period 1983 to 1993, all papers presented at the meetings in person were reviewed, while for the other years all papers included in the abstract lists (and/or the whole papers) were taken into account. For these other years there could be a very small overestimation-it could be that in some exceptional cases, an abstract was submitted but that paper was not presented.

The review process was undertaken at different points in time and by different groups of researchers. However, the principal investigator was the same on all occasions, and in all cases the same procedures have been followed.

When a study like this might be repeated in the future, new categories should be considered. Despite the limitations of this methodology, thematic coding and analysis of the papers presented at KBS symposia provide alcohol researchers with insight into the methods, progress, and priorities of the field. The research community can use the results to self-evaluate the scope and reach of previous meetings in relation to their goals on knowledge sharing in the field

\section{References}

Demers, A., Garretsen, H., Room, R., Rossow, I., \& Ugland, T. (2004). Kettil Bruun Society for Social and Epidemiological Research on Alcohol. Addiction, 2 (99), 161-164.

Garretsen, H.F.L., van Oers, J.A.M., \& van de Goor, L.A.M. (1993, June). The Kettil Bruun Society, the past decade: About booze: What do we choose? Talk conducted at the $19^{\text {th }}$ Annual Symposium, Kettil Bruun Society for social and epidemiological research on alcohol, Krakow, Poland.

Garretsen, H.F.L., Bongers, I.M.B., van de Goor, L.A.M., \& Roeg, D.P.K. (2003, June). The Kettil Bruun Society, the past decade: About booze: What do we choose? Another ten years later. Talk conducted at the $29^{\text {th }}$ Annual Symposium, Kettil Bruun Society for social and epidemiological research on alcohol, Krakow, Poland.

Garretsen. H., Audenaerdt, M., van de Goor, I., Roeg, D., van der Sar, R., Schoenmakers, T., \& van de Mheen, D. (2012), Social and epidemiological research on alcohol: Research presented at the meetings of the Kettil Bruun Society between 1983 and 2010. The International Journal of Alcohol and Drug Research, 1, 1-16. 